\title{
Hyperhydricity and Flavonoid Content of Scutellaria Species In Vitro on Polyester-supported Liquid Culture Systems
}

\author{
Ayse Tascan and Jeff Adelberg ${ }^{1}$ \\ Department of Horticulture, Clemson University, Clemson, SC 29634
}

\author{
Mevlut Tascan \\ School of Material Science and Engineering, Clemson University, Clemson, \\ $S C$
}

\section{Agnes Rimando}

USDA-ARS, Thad Cochran Research Center, University, MS

\section{Nirmal Joshee and Anand K. Yadav \\ Agricultural Research Station, Fort Valley State University, Fort Valley, GA}

Additional index words. skullcap, baicalin, baicalein, flavonoid, polyester fiber, medicinal plant, wogonin

\begin{abstract}
Three Scutellaria species (Scutellaria lateriflora, S. costaricana, and S. baicalensis) were grown in different in vitro physical environments: agar, liquid culture, and liquid culture with fiber-supported paper (with initial media volumes of $20 \mathrm{~mL}$ and $30 \mathrm{~mL}$ ). During an 8-week time course, tissue growth was assessed for each species by fresh weight (FW), dry weight (DW), percent DW, and multiplication ratio. Water use and hyperhydricity were also compared. Scutellaria lateriflora plantlets grown in liquid were hyperhydric despite the greatest accumulation of dry mass, but multiplication diminished with time as plants became hyperhydric. In contrast, $S$. costaricana and $S$. baicalensis plantlets had higher FW and DW on agar. With all Scutellaria species tested, plantlets grown on agar or fiber-supported paper were not hyperhydric, and fiber-supported paper with $20 \mathrm{~mL}$ initial volume yielded plants with the greatest percent DW. The lowered hyperhydricity was related to reduced water uptake. The flavonoids baicalin, baicalein, and wogonin were quantified in plants grown on fiber-supported paper culture. The baicalin concentrations in in vitro cultured $S$. lateriflora shoots was comparable to those of field-grown plants. The in vitro method presented a unique opportunity to enhance baicalein content and produce wogonin-rich roots. $S$. costaricana plantlets in vitro showed high levels of the three flavonoids compared with $\boldsymbol{S}$. baicalensis and $\mathbf{S}$. lateriflora. Growing non-hyperhydric tissues on fiber-supported paper, in vitro, allowed the clonal propagation of Scutellaria species with increased flavonoid content to proceed in a simple, controlled environment.
\end{abstract}

Scutellaria, a genus in the Lamiaceae family, has over 300 species with many known as medicinal plants in East Asia, Europe, the Middle East, and North America (Awad et al., 2003; Cole et al., 2007). Scutellaria baicalensis is named "skullcap," which describes the shape of the calyx at the base of flowers, which resembles miniature medieval helmets (Joshee et al., 2002). Scutellaria lateriflora is native to North America, has flavonoid-rich root, and has been used as an antidepressant (Duke, 2002). Scutellaria costaricana is native to Costa Rica and has been used as an

Received for publication 22 Oct. 2009. Accepted for publication 29 Jan. 2010.

We thank USDA-CSREES Research project 200202989 (Award \# 2002-38814-12612 to Fort Valley State University) for supporting this research.

${ }^{1}$ To whom reprint requests should be addressed; e-mail jadlbrg@clemson.edu. ornamental plant, but recent findings have shown it as a good source of bioactive flavonoids (Parajuli et al., 2009). Scutellaria baicalensis is native to China and Korea and has been used as an anticancer agent besides its ethnic use to treat bronchitis, hepatitis, diarrhea, and tumors (Joshee et al., 2002).

The amount of skullcap harvested and sold in world markets increased 2.5 times from $\approx 6,364 \mathrm{~kg}$ in 1997 to $15,910 \mathrm{~kg}$ in 2001 (Greenfeild and Davis, 2004). The annual consumption of skullcap increased by $23 \%$ between 2000 and 2001. Skullcap is currently recommended as an alternative sedative treatment to kava, which could increase the demand for this herb. The price of skullcap has steadily risen and is currently $\approx \$ 8.80$ to $\$ 17.60 / \mathrm{kg}$ U.S. Organically grown skullcaps fetch premium prices, for example, $\$ 17.60$ to $\$ 33.00 / \mathrm{kg}$ U.S. in Canada (Porter, 2006). The demand for skullcap in the world markets is predicted to grow at an annual rate of
$20 \%$ to $30 \%$ (Greenfeild and Davis, 2004). During $2001, \approx 70 \%$ of the world market demand for skullcap was supplied by sources outside of North America.

Tissue culture procedures have been used to propagate and distribute Scutellaria germplasm, including rare species, and to characterize secondary metabolism (Cole et al., 2007). Hyperhydricity is a physiological disorder frequently affecting shoots vegetatively propagated in vitro (Tascan et al., 2007). Hyperhydric shoots are characteristically translucent as a result of chlorophyll deficiency, poorly developed cell walls, and high water content. Hyperhydricity can be a major problem when in vitro shoots absorb excess water from the culture environment (Ziv, 2005). Hyperhydricity may arise from high humidity, low light intensity, high concentration of plant growth regulators, and tissue wounding. Also, differences in support material such as reduced agar concentration (from $0.8 \%$ to $0.58 \%$; Saher et al., 2004), switching agar to gelrite (Franck et al., 2004), or switching agar to liquid may result in more hyperhydric plantlets.

Different physical interventions have been explored to prevent hyperhydricity in liquid culture. For instance, sealing the culture vessels with a fluorocarbon polymer film eliminated hyperhydricity of Ixora coccinea L. plantlets (Lakshmanan et al., 1997). Rice plantlets grown in an immobilized culture system using polyurethane foam support cubes had longer shoots compared with plantlets cultured in liquid suspension. Liu et al. (2001) concluded that use of polyurethane reduced labor and time to propagate rice plantlets. There are many hurdles in the commercialization of desired genotypes using plant tissue culture; recalcitrance, low multiplication rate, slow growth in the medium, and hyperhydricity are some of these. Trying new growth regulators, alternate culture systems, and altering the culture vessel atmosphere are a few of the tools to maximize production of healthy plants.

We previously observed in vitro shoots of three Scutellaria species (S. lateriflora, $S$. costaricana, and $S$. baicalensis) had greater biomass when grown in liquid culture than on agar (Tascan et al., 2007). Hyperhydricity was observed with the microshoots immersed in liquid, with and without agitation, but with a floating paper culture system that was plant quality improved. In the current study, we refined the floating paper culture system by having paper layered over a polyester fiber matrix to control water availability and investigated the effect on the growth of three Scutellaria species. Biomass [fresh weight (FW) dry weight (DW), number of nodes] and plant quality (hyperhydricity, \%DW) were measured for $S$. lateriflora, $S$. costaricana, and $S$. baicalensis on agar, liquid, and the fibersupported paper. Flavonoid concentration was quantified for plantlets grown on fibersupported paper.

\section{Materials and Methods}

Culture conditions. Scutellaria baicalensis, $S$. costaricana, and S. lateriflora Stage II 
plantlets were provided by N. Joshee at the Agricultural Research Station of the Fort Valley State University, Fort Valley, GA. Plantlets were cultured in Murashige and Skoog semisolid medium (Murashige and Skoog, 1962) (4.33 g. $\mathrm{L}^{-1}$ MS salts M524; PhytoTechnology Laboratories, Shawnee Mission, KS) supplemented with $2 \mathrm{mg} \cdot \mathrm{L}^{-1}$ glycine (free base), $100 \mathrm{mg} \cdot \mathrm{L}^{-1}$ myo-inositol, 0.5 $\mathrm{mg} \cdot \mathrm{L}^{-1}$ nicotinic acid (free acid), $0.5 \mathrm{mg} \cdot \mathrm{L}^{-1}$ pyridoxine $\cdot \mathrm{HCL}, 0.1 \mathrm{mg} \cdot \mathrm{L}^{-1}$ thiamine $\cdot \mathrm{HCl}$, benzyladenine $(5 \mu \mathrm{M}), \alpha$-naphthalene acetic acid $(0.025 \mu \mathrm{M}), 30 \mathrm{~g} \cdot \mathrm{L}^{-1}$ sucrose, and $5 \mathrm{~g} \cdot \mathrm{L}^{-1}$ agar (bacteriological grade; Prod. No. A296; PhytoTechnology Laboratories). The culture medium was autoclaved at $121^{\circ} \mathrm{C}$ for $40 \mathrm{~min}$. Plantlets were transferred to $180-\mathrm{mL}$ cylindrical glass vessels containing $30 \mathrm{~mL}$ MS semisolid medium with six nodes per jar. Uniform size plantlets were selected and subcultured every 6 weeks.

Fiber preparation. Needle-punched nonwoven fabric matte was produced and provided by Dr. Mevlut Tascan of the Material Science and Engineering Department at Clemson University, Clemson, SC. Round polyester fiber (75\% recycled, $25 \%$ copolyester) was used (Table 1). Premixing was achieved by the CMC Rando Cleaner (Model No. RC-24; Carolina Machinery; fiber mixing and cleaning machine). Then, fibers were fed through a chute to the $50-\mathrm{cm}$ Bematic Card (Costruzione Machine Tessili, Bamatic Mettarini and Serafini Sr, Prato, Italy), and the web was fed onto a $60 \mathrm{~cm}$ Automatex cross lapper conveyor system (Impianti Automatex Nonwoven, Saba, Italy). The fabric was then needle-punched by the Automatex Needle Punching (Impianti Automex Nonwoven; $\approx 50$ strokes/min) for handling purposes; then this lightly needlepunched, nonwoven fabric was needled again at $\approx 250 \mathrm{strokes} / \mathrm{min}$. The resulting needlepunched samples had a density of $\approx 150 \mathrm{~g} \cdot \mathrm{m}^{-2}$. The fibers were cut into uniform sizes $(5 \mathrm{~cm} \times$ $5 \mathrm{~cm}$ ), rinsed with distilled water, and dried before putting into the vessels.

Physical effects experiment. Plantlets were cultured on four different culture conditions depending on the physical nature of the support: 1) agar gel (AG) culture; 2) liquid stationary (LS) culture; 3) liquid culture with polyester fiber supported paper (FS) having $30 \mathrm{~mL}$ initial media (FS30); and 4) FS having $20 \mathrm{~mL}$ initial media (FS20). The agar medium was prepared as described previously. The liquid media was prepared similar to the agar media, except that agar was omitted. Each vessel contained six nodes of each species placed in Magenta GA7 (Magenta Corp., Chicago, IL) vessels with $30 \mathrm{~mL}$ initial volume for the AG, LS, and FS30. In FS20, $20 \mathrm{~mL}$ initial volume was used and $5 \mathrm{~mL}$ of medium was added on the fourth and the sixth weeks. The fiber-supported paper system consisted of a polyester fiber matte (Clemson University, Clemson, SC), $5 \mathrm{~cm} \times 5 \mathrm{~cm}$, placed under the seed germination paper (Anchor Paper Co., St. Paul, MN), $5 \mathrm{~cm} \times 5 \mathrm{~cm}$, in the Magenta GA7 boxes. Plant nodes were placed on the seed germination paper. Plantlets were cultured at $25 \pm 2{ }^{\circ} \mathrm{C}$ under fluorescent lights with an intensity of 25 to $30 \mu \mathrm{mol} \cdot \mathrm{m}^{2} \cdot \mathrm{s}^{-1}$ photosynthetically active radiation for $16 \mathrm{~h} \cdot \mathrm{d}^{-1}$. Plantlets were harvested in the second, fourth, sixth, and eighth weeks. The experiment was conducted in a completely randomized design with four treatments and three replicates. Plantlets were blotted on a paper towel, and the FW, DW, hyperhydricity, and residual sucrose concentration were determined. Hyperhydricity was observed by visual assessment using a 1 to 5 numerical scale (Fig. 1). The concentration of residual sucrose was obtained by using a hand refractometer (Atago, Tokyo, Japan) and calculated as \% Brix (grams sucrose per $100 \mathrm{~mL}$ medium). Plantlets were placed in paper envelopes and dried at $80{ }^{\circ} \mathrm{C}$ for $48 \mathrm{~h}$. The DW was then measured. Additional calculations were performed as follows:

\section{Multiplication ratio \\ $=($ no. of nodes at harvest $) /$}

(initial no. of nodes)

Percent dry weight (\%DW)

$=($ dry weight $/$ fresh weight $) \times 100$
Percentage sucrose use

$=\{1-[($ residual sucrose amount

$\times$ residual volume amount)/

(initial sucrose amount

$\times$ initial volume amount) $]\} \times 100$

\section{Water use in tissue per plant}

$$
=(\mathrm{FW}-\mathrm{DW}) / \text { no. of nodes at harvest }
$$

Extraction and analysis of flavonoids. Dried and powdered tissues of the three species that were grown for 8 weeks on a scaled-up FS system $(25$ nodes with $150 \mathrm{~mL}$ of medium in $110 \mathrm{~mm} \times 297 \mathrm{~mm}$ Southern Sun Vessels; Southern Sun BioSystems, Hodges SC) were extracted using an $\mathrm{ASE}^{\circledR}$ apparatus (Dionex Corporation, Sunnyvale, CA). Ten-gram samples (two samples of each species) were mixed with purified sand (Fisher Scientific, Pittsburgh, PA) and loaded in the extraction cartridges. Extraction was carried out under the following conditions: heat, $5 \mathrm{~min}$; static, $10 \mathrm{~min}$; flush volume, $100 \mathrm{~mL}$; purge, $90 \mathrm{~s}$; pressure, $1000 \mathrm{psi}$; temperature, $40{ }^{\circ} \mathrm{C}$; extraction solvent, methanol:water $(80: 20)$, four cycles. The extracts were concentrated

Table 1. Different fiber characteristics for the nonwoven fabrics used in tissue culture experiments.

\begin{tabular}{lclcc}
\hline Fiber $(\%)$ & Diam $(\mu \mathrm{m})$ & \multicolumn{1}{c}{ Type } & Cross-section & Length \\
\hline 25 & 20 & Bicomponent polyester fiber & Round & $1.5^{\prime \prime}$ \\
75 & 40 & Orange polyester fiber & Round & $2^{\prime \prime}$ \\
\hline
\end{tabular}
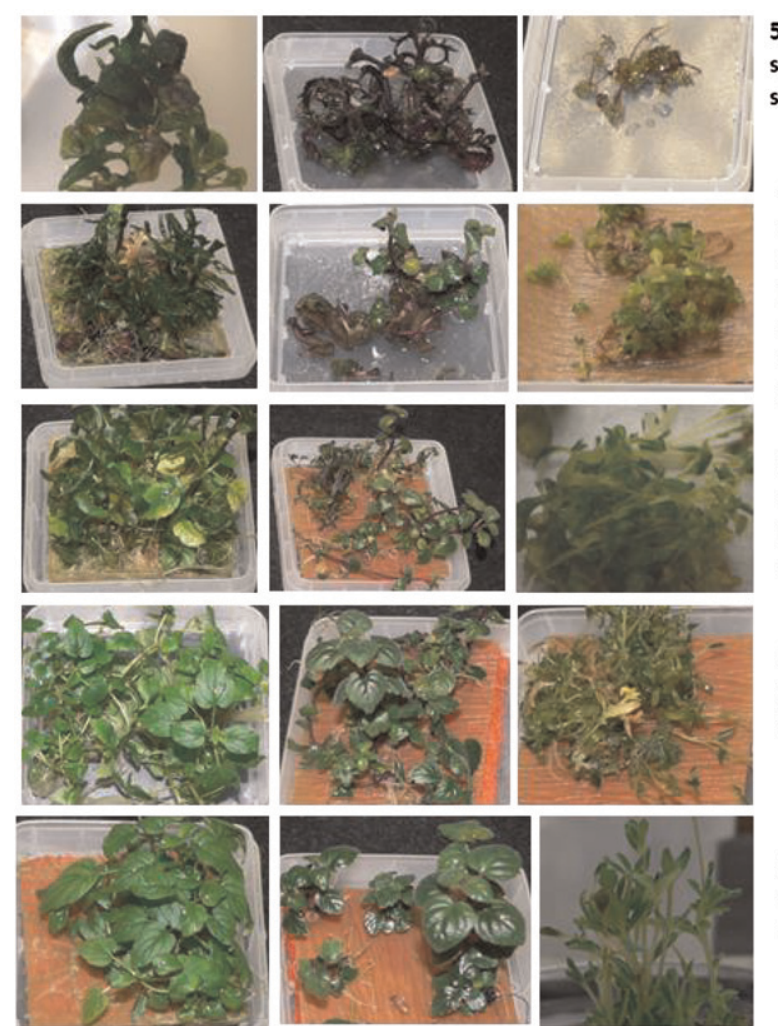

\section{S. lateriflora}

S. costaricana

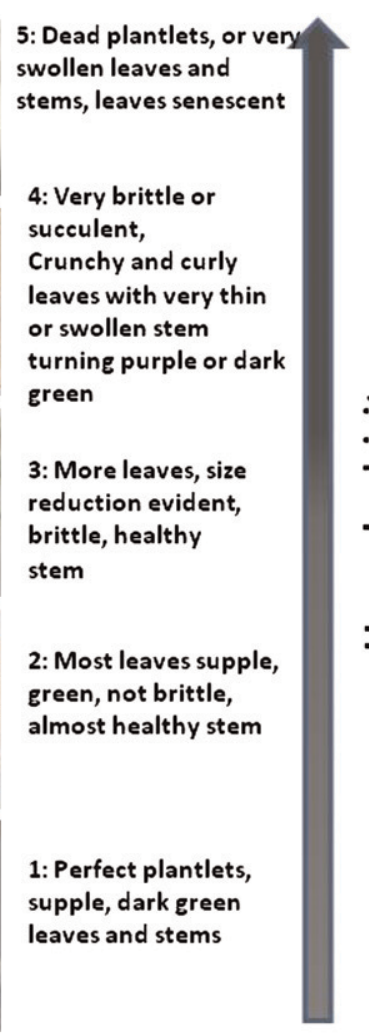

S. baicalensis

Fig. 1. Hyperhydricity scale for Scutellaria species (S. lateriflora, A; S. costaricana, B; S. baicalensis, C). Scaling was based on visual observation. 
under vacuum using a Savant SpeedVac (Model SPD121P; Savant Instruments, Inc., Holbrook, NY). Dried extracts were weighed and dissolved in $0.5 \% \mathrm{HCl}$-methanol and analyzed by high-performance liquid chromatography (HPLC) for their flavonoid content. The HPLC method has been described in detail (Parajuli et al., 2009).

Statistical analysis. Data were analyzed with SAS 9.1 (SAS Institute, Cary, NC) and means were separated by using Fisher's least significant difference $(\alpha=0.05)$. The time course was analyzed as a factorial (physical environment $\times$ time) with significant linear and quadratic models prepared for each physical treatment and each species.

\section{Results and Discussion}

Plant growth and hyperhydricity. The three Scutellaria species grew differently in the various physical environments. However, plantlets of the three species had similar response in terms of hyperhydricity and \%DW. Plantlets of all species grown in the LS culture were hyperhydric and had the smallest multiplication ratio. However, the plantlets grown on FS and AG culture were less hyperhydric. A subjective scale for hyperhydricity was established for Scutellaria species. Figure 1 presents plantlets showing the range of hyperhydricity scored on a numerical scale of 1 to 5 ( 5 being the most hyperhydric).

Plantlets of $S$. lateriflora grown in LS culture had the greatest FW (Fig. 2A) and
DW (Fig. 2B) for the entire 8-week period, although growth had slowed in the sixth week. The FW of plantlets grown in $\mathrm{AG}$ and FS cultures increased at a constant rate for the entire 8 weeks. The LS plantlets had significantly greater FW and DW at week 8 compared with AG and FS plantlets. Percent DW of the plantlets was significantly greater in FS20 compared with other cultures (Fig. 2C). However, although \%DW and hyperhydricity were improved in FS20 culture, it produced the smallest biomass (FW, DW).

Scutellaria lateriflora plantlets grown in FS and AG cultures were not hyperhydric after 8 weeks in culture compared with plantlets grown in the LS culture (Fig. 2E). Immersing the plantlets in the media caused hyperhydricity and these hyperhydric plantlets used the most sucrose (data not shown) and the most water (Fig. 2F), similar to our previous work (Tascan et al., 2007). The plantlets of $S$. lateriflora had a greater multiplication ratio in AG than in FS culture by the end of week 8 (Fig. 2D). Multiplication of LS plantlets declined over time, which may be related to slowing biomass accumulation. The decreased multiplication ratios might be the result of hyperhydricity. The hyperhydric plants in the LS culture accumulated ample DW.

Scutellaria lateriflora plantlets in the FS20 culture had greater $\% \mathrm{DW}$, were less hyperhydric, had a relatively high multiplication ratio, and used the least water per plantlet. The growth conditions of FS30 and FS20 cultures differed during the first 4 weeks. The lower initial media volume used for the FS20 culture increased the vertical path the liquid must follow from the liquid reservoir through the fiber matte to the plantlets and reduced the interface surface meniscus between the media and the plantlets. Decreased initial volume resulted in increased $\%$ DW and decreased FW. Fiber makes water available to the plant through a capillary action and the restricted water flow prevents hyperhydricity. Plantlets grown in the liquid culture had high DW, but also absorbed large amounts of water and became hyperhydric.

The plantlets of $S$. costaricana in AG culture had the greatest FW as well as DW (Fig. 3A-B). Scutellaria costaricana plantlets in FS had significantly greater $\% \mathrm{DW}$ for the entire 8-week period (Fig. 3C) compared with AG- and LS-grown plantlets. Additionally, the $S$. costaricana plantlets in FS and AG cultures had a higher multiplication ratio compared with plantlets in the LS culture at the end of 8 weeks (Fig. 3D). The plantlets grown on FS cultures were less hyperhydric (Fig. 3E) compared with the AG plantlets, which had intermediate hyperhydricity and had their best appearance at $\approx 6$ weeks. Plantlets in LS culture were the most hyperhydric for the entire 8-week period. LSgrown plantlets used the most water, and the FS used the least water (Fig. 3F).

We hypothesized that plantlets that absorbed excess water would suffer from hypoxia, which is a metabolic stress related to reduced growth. $S$. costaricana plantlets in
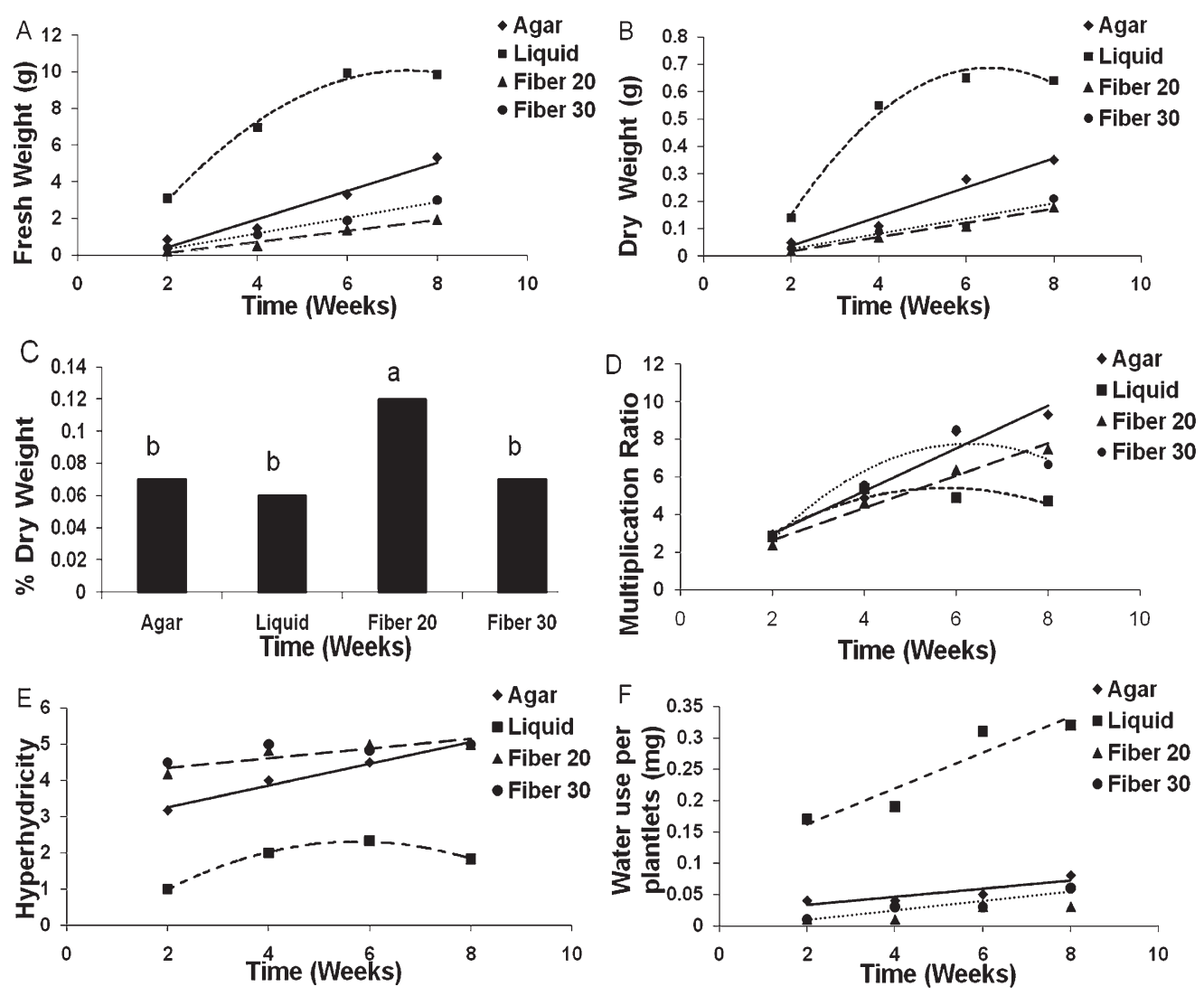

Fig. 2. Scutellaria lateriflora response [fresh weight (A), dry weight (B), percent dry weight $(\mathbf{C})$, multiplication ratio (D), hyperhydricity (E), and water use per plantlet $(\mathbf{F})$ in agar, a fiber system with 20 and $30 \mathrm{~mL}$ initial medium volume, and liquid cultures during 8 weeks. Only significant linear fits were presented. 

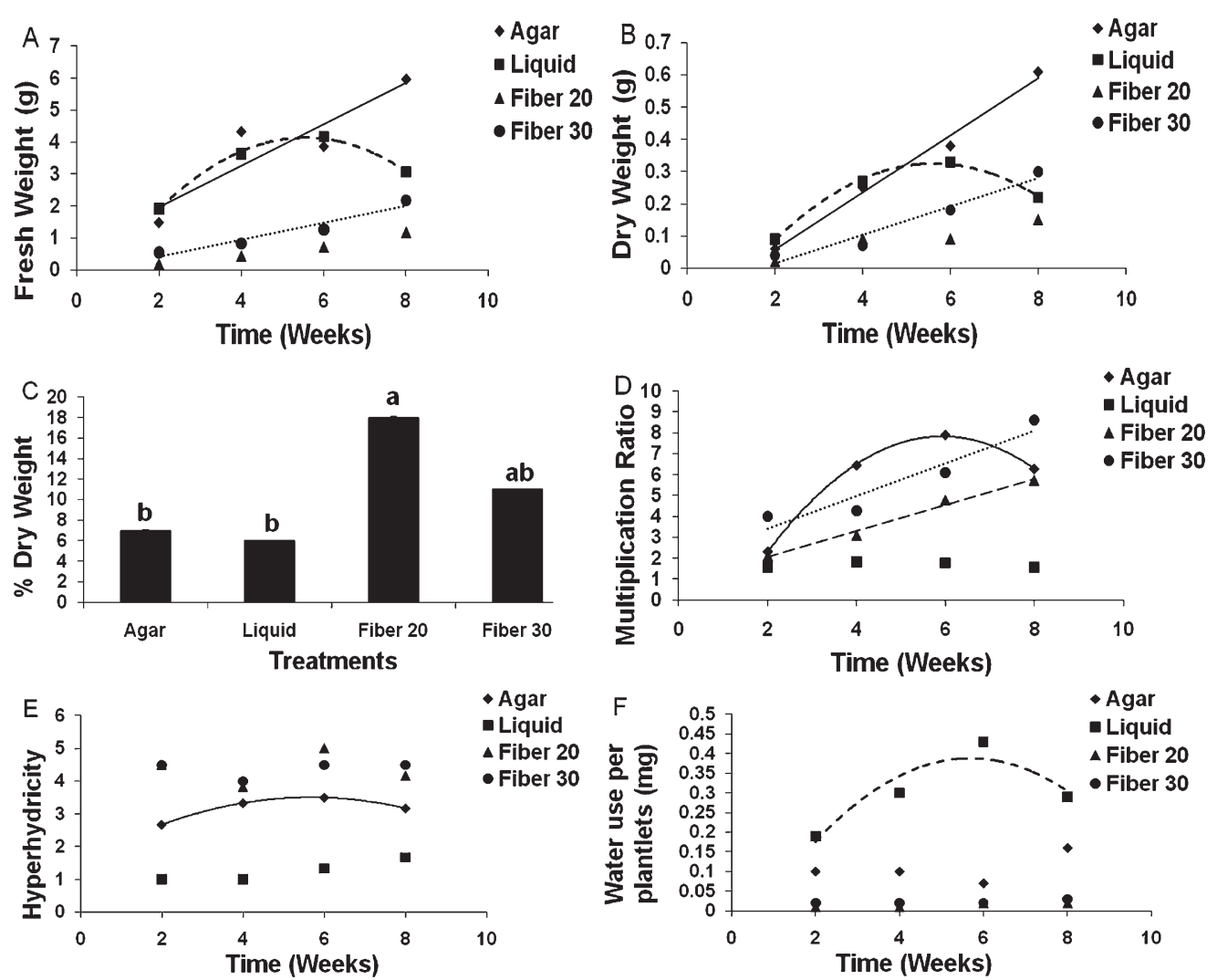

Fig. 3. Scutellaria costaricana response [fresh weight (A), dry weight (B), percent dry weight (C), multiplication ratio (D), hyperhydricity (E), and water use per plantlet (F)] in agar, a fiber system with 20 and $30 \mathrm{~mL}$ initial medium volume, and liquid cultures during 8 weeks. Only significant linear fits were presented.
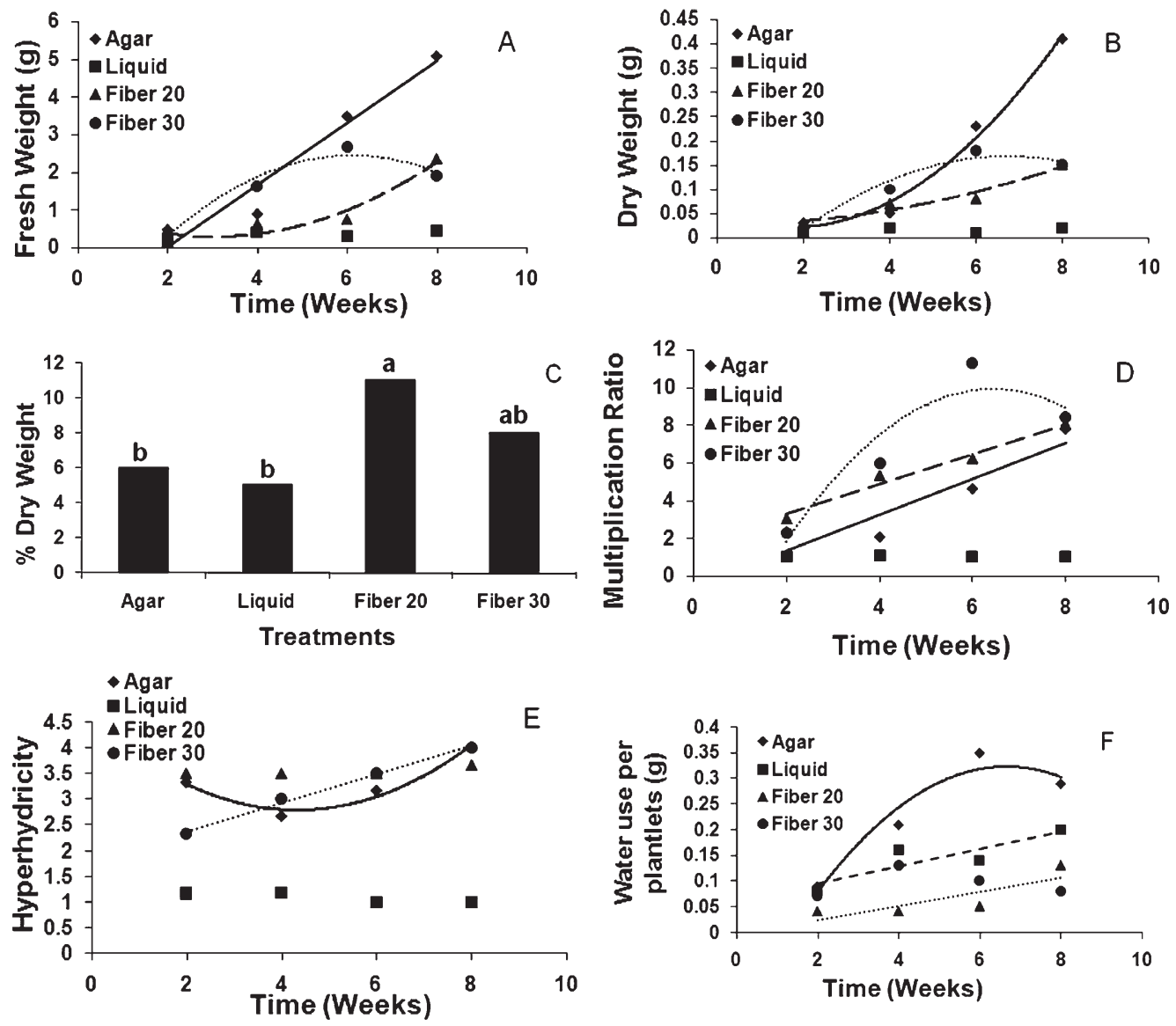

Fig. 4. Scutellaria baicalensis response [fresh weight (A), dry weight (B), percent dry weight (C), multiplication ratio (D), hyperhydricity (E), and water use per plantlet (F)] in agar, a fiber system with 20 and $30 \mathrm{~mL}$ initial medium volume, and liquid cultures during 8 weeks. Only significant linear fits were presented. 
the LS culture caused tanning of the media, which may be the result of phenolic exudates produced by the plantlets under stress. Similarly, S. lateriflora plantlets from LS culture became more hyperhydric. The fiber matrix may have reduced hyperhydricity by reducing the flow of water to the plantlets.

Scutellaria baicalensis plantlets in AG culture yielded the greatest FW for the entire 8 weeks (Fig. 4A). The LS culture plantlets stopped growing when immersed in liquid and had the lowest FW. The DW of the plantlets was similar to the FW (Fig. 4B). Plantlets in FS20 had significantly greater $\% \mathrm{DW}$ followed by plantlets in the FS30 for the entire 8-week duration (Fig. 4C). The AG and LS culture plantlets had low \%DW. The plantlets of $S$. baicalensis in AG and FS had similar multiplication ratios at the end of 8 weeks (Fig. 4D). Plantlets in LS culture became hyperhydric and did not multiply or grow. The plantlets of $S$. baicalensis in the FS and AG cultures were the least hyperhydric for the entire 8 weeks (Fig. 4E).

Scutellaria baicalensis plantlets in $\mathrm{AG}$ culture used the most water for the entire 8 weeks (Fig. 4F). Callus production was observed for $S$. baicalensis plantlets in the AG culture. There was no callus apparent in the FS cultures. FS20 plantlets used less water compared with AG culture plantlets and these plantlets had significantly higher $\% \mathrm{DW}$ for the entire 8 weeks.

Flavonoid content. Plants grown on a scaled-up FS system (Fig. 5) were analyzed for the three major Scutellaria flavonoids: baicalin, baicalein, and wogonin (Table 2). For S. costaricana and S. baicalensis, root and shoot samples were pooled to obtain $10 \mathrm{~g}$ of tissue dry weight needed for extraction.

Shoots of plantlets of $S$. lateriflora grown in the scaled-up FS culture system had baicalin and wogonin concentrations (Table 2) similar to values reported for leaves and stems of field-grown plants (Nishikawa et al., 1999). Baicalein was not detected; however, we found this flavonoid in the root and shoots of $S$. lateriflora FS plantlets. The wogonin concentration in $S$. lateriflora roots was notably high (Table 2), similar to those obtained in tissue cultures by Nishikawa et al. (1999). Field-grown $S$. lateriflora roots are too thin and fibrous to be efficiently harvested from the soil. Therefore, in vitro culture of S. lateriflora may provide a convenient method to obtain root biomass as a rich source of wogonin. A representative chromatogram is shown in Figure 6.

We recently observed $S$. costaricana to be a good source of bioactive flavonoids (Parajuli et al., 2009). The extract of the mixed shoot and root preparation of $S$. costaricana from the FS system (Table 2) had baicalin and bacialein concentrations similar to those of a physical mixture of the roots, stems, and leaves of greenhouse-grown plants. Wogonin concentration was higher in greenhouse-grown plants than the tissue-cultured plantlets.

Other studies have shown that field-grown S. baicalensis (Tani et al., 1985) had flavonoid concentrations similar to those (Zobayed et al.,
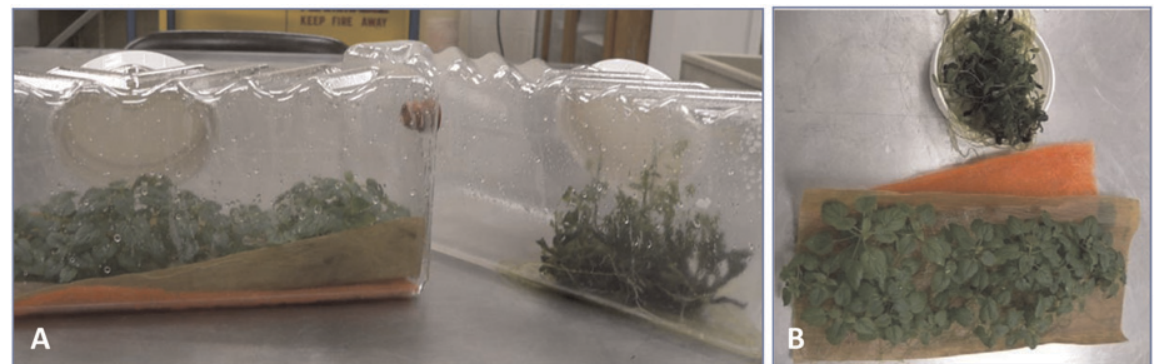

Fig. 5. Plantlets of Scutellaria lateriflora after 8 weeks of growth in $150 \mathrm{~mL}$ of medium in Southern Sun Vessels with and without polyester fiber support. Plants without fiber were completely hyperhydric.

Table 2. Flavonoid contents of plantlets after 8 weeks of growth on fiber-supported paper treatment $\left(\mu \mathrm{g} \cdot \mathrm{mg}^{-1}\right.$ tissue dry weight $\left.\pm \mathrm{SE}\right)$.

\begin{tabular}{llccr}
\hline Species & \multicolumn{1}{c}{ Organ } & Baicalin & \multicolumn{1}{c}{ Baicalein } & \multicolumn{1}{c}{ Wogonin } \\
\hline S. lateriflora & Root & $0.639 \pm 0.035$ & $0.104 \pm 0.000$ & $1.262 \pm 0.005$ \\
S. lateriflora & Shoot & $1.071^{z}$ & $0.419^{z}$ & $0.524 \pm 0.107$ \\
S. baicalensis & Root and shoot & $0.418 \pm 0.007$ & $0.107 \pm 0.002$ & $0.0278 \pm 0.001$ \\
S. costaricana & Root and shoot & $1.567 \pm 0.068$ & $0.734 \pm 0.106$ & $0.208 \pm 0.030$ \\
\hline
\end{tabular}

${ }^{\mathrm{z}}$ Values are from a single sample.
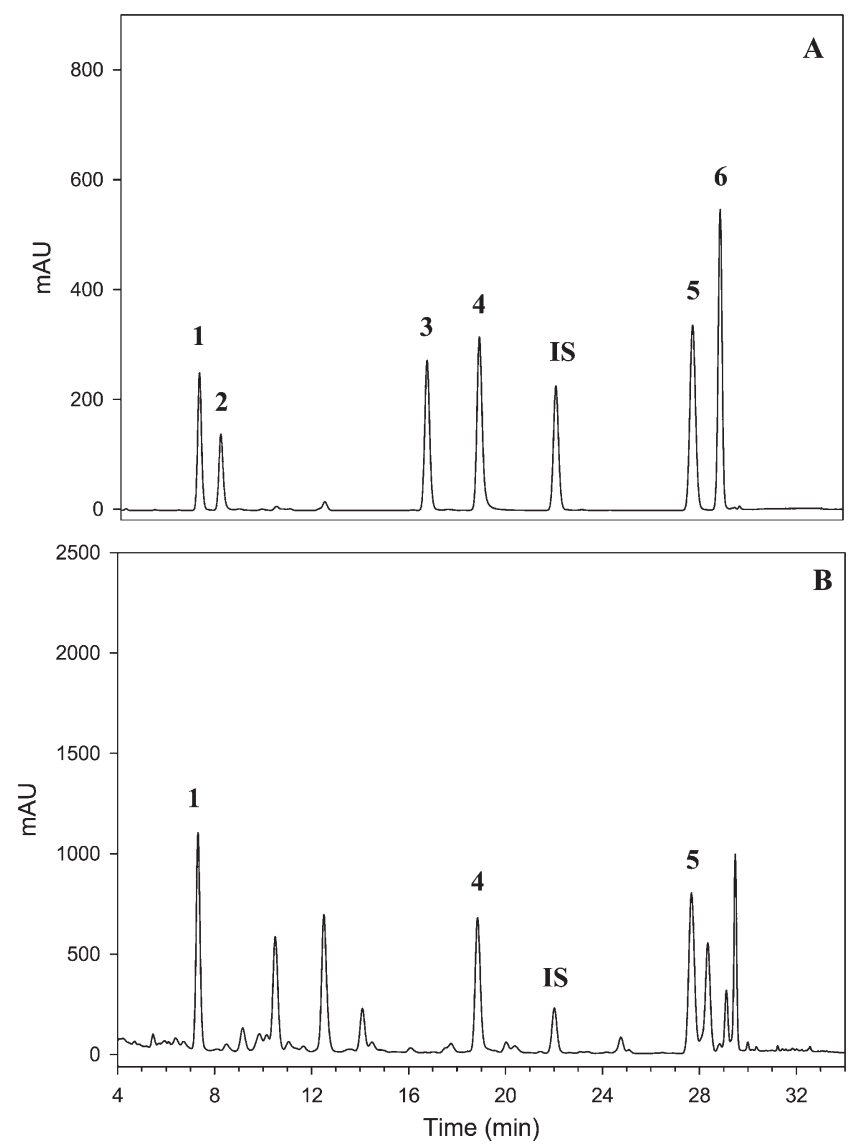

Fig. 6. High-performance liquid chromatography trace of (A) flavonoid standards: 1) baicalin, $7.2 \mathrm{~min}$; 2) scutellarein, $8.1 \mathrm{~min}$; 3) apigenin, $16.7 \mathrm{~min}$; 4) baicalein, $18.9 \mathrm{~min}$; IS, internal standard 6hydroxyflavone, $22.0 \mathrm{~min}$; 5) wogonin, $27.7 \mathrm{~min}$; chrysin, $28.9 \mathrm{~min}$. (B) Extract of Scutellaria lateriflora shoots. Peaks were identified from their retention time and ultraviolet spectra.

2004) that were produced on the agar medium under the $\mathrm{CO}_{2}$-enriched forced air ventilation. In our study, the flavonoid concentration of the combined shoots and roots grown on FS system was $\approx 1 / 10$ the concentration found in the shoots of $S$. baicalensis grown in a forced ventilation $\left(\mathrm{CO}_{2}\right.$-enriched) agar-containing vessel and two- to fivefold less than those grown in liquid bioreactors (Zobayed et al., 2004). However, our values are within ranges reported for 26 tissue-cultured $S$. baicalensis germplasm lines, which showed a wide range 
of flavonoid content: from 0.42 to 4.52 $\mu \mathrm{g} \cdot \mathrm{mg}^{-1}$ baicalin, 0.08 to $3.13 \mu \mathrm{g} \cdot \mathrm{mg}^{-1}$ baicalein, and 0.002 to $0.064 \mu \mathrm{g} \cdot \mathrm{mg}^{-1}$ wogonin (Murch et al., 2004). The advantage of the fiber-supported paper used in the present investigation is that it is mechanically simpler than the bioreactors used by Zobayed et al. (2004).

This is the first observation that the principal Scutellaria flavonoids (baicalin, baicalein, and wogonin) are being produced in FS plantlets at reasonable levels. Plant organ bioreactor systems allow cultivation where it may be difficult to cultivate in the open field (e.g., S. lateriflora roots). Also, plants that have not been well characterized (e.g., S. costaricana) may be compared, under controlled conditions, with other better-characterized Scutellaria species. There are dozens of Scutellaria species that are yet to be analyzed.

The success of the FS systems was the production of plantlets that are generally not hyperhydric and had relatively high multiplication ratios. The fiber reduced water uptake and prevented hypoxia. The fiber matrix may be constructed with varying thickness or used with varied volumes of medium to allow greater biomass with reduced hyperhydricity. The simple alterations of medium volume between the FS30 and FS20 showed some of the flexibility and plant responses this system allowed. Because the fiber can be cut to any size, scale-up in two dimensions looks like a question of the ease of materials handling. Compared with a greenhouse, the closed tissue culture system gives greater control (environmental, pests, and pesticides).

The fiber system was not optimized for phytochemistry, and this reported assay followed the first set of scaled-up trials. Further experiments on phytochemical optimization of greenhouse or bioreactor scale-up had not been conducted. Process optimization would include refining the physical environment and selection of plant species for increased biomass and flavonoid concentrations. The
FS system is mechanically simple and was easily scaled to larger vessels, and so advantages of bioreactors may be achieved in an economic and robust platform. A second advantage over greenhouse or soil is that flavonoids produced in fine feeder roots of $S$. lateriflora (like wogonin) may be enhanced by encouraging root mass with compounds such as auxins. Experiments on phytochemical optimization of FS bioreactor scale-up had not been conducted.

Keeping plantlets in the gaseous phase (i.e., FS and AG systems) resulted in better quality plantlets (reduced hyperhydricity) with a higher multiplication ratio compared with plantlets completely immersed in the liquid phase. Fiber creates a matrix that reduces media uptake, prevents hyperhydricity, and may help to improve the qualities of the plant biomass.

\section{Literature Cited}

Awad, R., T. Arnason, V. Trudeau, C. Bergeron, J.W. Budzinski, B.C. Foster, and Z. Merali. 2003. Phytochemical and biological analysis of skullcap (S. lateriflora L.): A medicinal plant with anxiolytic properties. Phytomedicine 10: 640-649.

Cole, I., P. Saxena, and S. Murch. 2007. Medicinal biotechnology in the genus Scutellaria. In Vitro Cell Dev Biol Plant. 43:318-327.

Duke, A.C. 2002. Handbook of medicinal herbs. CRC Press, Boca Raton, FL. p. 50-673.

Franck, T., C. Kevers, T. Gaspar, J. Dommes, C. Deby, R. Greimers, D. Serteyn, and G. DebyDupont. 2004. Hyperhydricity of Prunus avium shoots cultured on Gelrite: A controlled stress response. Plant Physiol. Biochem. 42:519-527.

Greenfeild, J. and J.M. Davis. 2004. Skullcap (Scutellaria laterifolia L.). Medicinal herb production guide. 12 July 2006. <http://www. naturalmedicinesofnc.org $>$.

Joshee, N., T.S. Patrick, R.S. Mentreddy, and A.K. Yadav. 2002. Skullcap: Potential medicinal crop, herbs, p. 22-30. In: Janick, J. and A. Whipkey (eds.). Medicinals and aromatics trends in new crops and new uses. ASHS Press, Alexandria, VA.
Lakshmanan, P., C.L. Lee, and C.J. Goh. 1997. An efficient in vitro method for mass propagation of a woody ornamental Ixora coccinea L. Plant Cell Rep. 16:572-577.

Liu, C., K. Moon, H. Honda, and T. Kobayashi. 2001. In situ regeneration of rice (Oryza sativa L.) callus immobilized in polyurethane foam. J. Biosci. Bioeng. 91:76-80.

Murashige, T. and F. Skoog. 1962. A revised medium for rapid growth and bio-assays with tobacco tissue cultures. Plant Physiol. 15:473497.

Murch, S.J., H.P.V. Rupasinghe, D. Goodenowe, and K. Saxena Praveen. 2004. A metabolomic analysis of medicinal diversity in Huang-qin (Scutellaria baicalensis Georgi) genotypes: Discovery of novel compounds. Plant Cell Rep. 23:419-425.

Nishikawa, K., H. Furukawa, T. Fujioka, H. Fuji, K Mihashi, K. Shimomura, and K. Ishmaru. 1999. Phenolics in tissue cultures of Scutellaria. Nat. Med. (Tokyo) 53:209-213.

Parajuli, P., N. Joshee, A. Rimando, S. Mittal, and A.K. Yadav. 2009. In vitro anti-tumor mechanisms of various Scutellaria extracts and constituent flavonoids. Planta Med. 75:41-48.

Porter, B. 2006. Skullcap production in Saskatchewan. 12 July 2006. <http://www.agr.gov.sk.ca/ docs/crops/horticulture/skullcap.asp>.

Saher, S., A. Piqueras, E. Hellin, and E. Olmos. 2004. Hyperhydricity in micropropagated carnation shoots: The role of oxidative stress. Plant Physiol. 120:152-161.

Tani, T., T. Katsuki, M. Kubo, and S. Arichi. 1985. Histochemistry VII. Flavones in Scutellaria Radix. Chem. Pharm. Bull. (Tokyo) 33:4894 4900.

Tascan, A., J.W. Adelberg, M. Tascan, N. Joshee, and A.K. Yadav. 2007. Liquid culture system for Scutellaria species. Acta Hort. 756:163-170.

Ziv, M. 2005. Simple bioreactors for mass propagation of plants, p. 79-95. In: Hvoslef-Edie, A.K. and W. Preil (eds.). Liquid culture systems for in vitro plant propagation. Springer, Doordecht, The Netherlands.

Zobayed, S.M.A., S.J. Murch, H.P.V. Rupasinghe, J.G. de Boer, B.W. Glickman, and P.K. Saxena. 2004. Optimized system for biomass production, chemical characterization and evaluation of chemo-preventive properties of Scutellaria baicalensis Georgi. Plant Sci. 167:439-446. 